\title{
Mechanical Performance of a Poly L Lactide Acid Device for Reconstruction of Fractured Metacarpal
}

\author{
D.A. Nunez ${ }^{1, *}$, A.K.N $\operatorname{Vargas}^{2}$, O.F. Aviles ${ }^{1}$ and M.F. Mauledoux ${ }^{1}$ \\ ${ }^{1}$ Mechatronics Engineering, Davinci Research Group, Universidad Militar Nueva Granada, Av Calle 100, Bogotá, Colombia. \\ ${ }^{2}$ Grupo de Investigación en Ingeniería Ambiental. Vicerrectoria de Investigaciones. Universidad Manuela Beltrán. Av. Circunvalar \\ No 60-00, Bogotá, Colombia.
}

Received 19 August 2018; Accepted 20 December 2019

\begin{abstract}
A fractured metacarpal joined with a poly $\mathrm{L}$ lactide acid device is studied analyzing its performance under different kinds of loads and studying them in a finite element analysis software. The metacarpal and polymer devices are designed in a computer aided design software. Relaxation and tensile performance of the polymer are determined. Then, we developed a rheological model. The rheological model is simulated and the chosen model with two Neo-Hookean networks in a parallel distribution lead to a fit of $98 \%$. Flexural and tensile performance of the assembly is depicted. The Von Mises stress is used as way to determine the correct performance of assembly. After the analysis, the holes in the bone is the main cause of failure in this fixation method because of the stress associated with accumulation of stress owing to change in geometry. Additionally, the use of finite element analysis reduces resources and destructive tests can be avoided. Furthermore, a mathematical model of the mechanical behavior of the poly-L-lactide acid is achieved using a Parallel Network Model.
\end{abstract}

Keywords: Poly L Lactide Acid, Parallel Model Material, Fractured Metacarpal.

\section{Introduction}

Metacarpal fractures are common orthopedic injuries and one of the most challenging to handle owing to the loads that the bone borne, age of patient, fracture configuration and type of fixation [1]. There are many procedures, techniques and fixation methods to stabilize displaced bone fractures such as surgeries and implants [2]. Among fixation devices are those made of metals. Formerly, those kinds of devices generated many complications [3]. Hence, biopolymer devices have been developed owing to their no toxicity, no necessary extra incision to remove them, and their mechanical response [4]. This class of polymers include poly glycolic acid (PGA), poly caprolactone (PCL), poly dioxanone (PDO) and poly lactide acid (PLA) among others [5]. Poly-L-lactide acid (PLLA) is a popular stereoisomer used as material of bone fixation material [6]. Device geometry, body location, and mechanical requirements are challenges related to the use of PLLA devices because that polymer has exhibited premature failure due to its viscoplastic behavior [4]. The device performance depends on the nonlinear mechanical behavior of PLLA; however, there is a lack of information about the mechanical behavior under physiological conditions of PLLA devices and metacarpal fractured under different kind of loads [7]. This work focusses on mathematical models and finite element analysis (FEA) to describe the mechanical response under tensile and flexural loads of a repairing device made of PLLA to fix metacarpal fracture. Besides, this paper presents the study of convergence of mesh density and the analysis of critical zone of repair.

*E-mail address: v7700100@unimilitar.edu.co

ISSN: 1791-2377 @ 2020 School of Science, HHU. All rights reserved. doi:10.25103/iestr.131.04

\section{Materials and Methods.}

\subsection{Polymer features.}

PLLA has the same features used in former work [7] and was provided by CORBION.

Mechanical performance of PLLA.

Mechanical properties of PLLA were determined following ASTM D638-10. Polymer specimens were tested under different conditions and varying strain rates between $0.05 / \mathrm{s}$ and $0.001 / \mathrm{s}$ and a relaxation behavior was also carried out and Fig. 1 and Fig. depict its stress strain behavior. The test followed previous reported protocol [7].

Next, the mathematical model which described the rheological behavior was selected based on previous studies. A parallel network model for rheological behavior with two networks was chosen based on its accurate to predict nonlinear viscoplastic behavior. Fig. depicts the configuration of the model. A Neo Hookean hyperlelastic behavior was described in the first network (Eq. 1) and a Neo Hookean hyperelastic behavior with a flow component, represented as a power flow with double exponential yield evolution (Eq. 2), was depicted in the second network. The values of shear modulus. The first step established stress strain relation from the experimental tests (Figure 1 and 2) and in the second step, the material model, taking into account hypereslastic behaviors and flow features were included. evolution (Eq. 3).

\subsection{Theory and Calculations}

The Nelder Mead Simplex algorithm method was used to obtain the material parameters. Two steps were followed for obtaining material parameters. The first step established stress strain relation from the experimental tests (Figure 1 and 2) 
and in the second step, the material model, taking into account hypereslastic behaviors and flow features were included. The mean square error between experimental data and material model was used as the objective function.

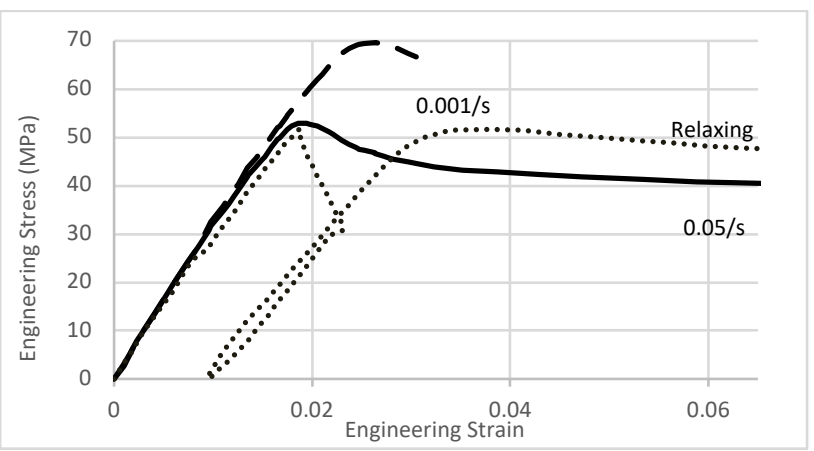

Fig. 1. Experimental tests varying strain rate and relaxing behavior.

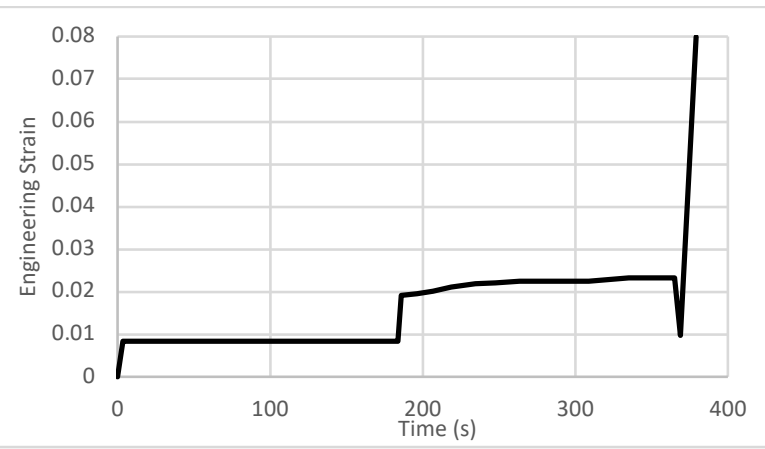

Fig. 2. Relaxing strain time behavior.

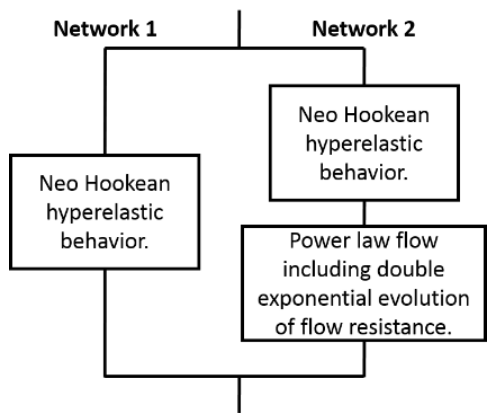

Fig. 3. Rheological representation of Parallel Network model.

$$
\sigma=\mu /|F| \operatorname{dev}\left(|F|^{-2 / 3} F F^{T}\right)+\kappa(|F|-1) I
$$

Where:

$$
\begin{array}{ll}
\sigma: & \text { Cauchy stress. } \\
\kappa: & \text { Bulk modulus. } \\
F: & \text { Force. } \\
\mu: & \text { Shear Modulus. } \\
F^{T}: & \text { Force transpose. } \\
I: & \text { Identical Matrix }
\end{array}
$$$$
f_{r}=\left[\tau /\left(R_{\tau} f_{p} f_{T} f_{E}\right)\right]^{m}
$$

Where:

$$
\begin{array}{ll}
f r: & \text { Flow rate } \\
R_{\tau}: & \text { Shear flow resistance. } \\
\tau: & \text { Shear stress. } \\
m: & \text { Shear flow exponent. }
\end{array}
$$

$f_{p}: \quad$ Pressure dependence factor $=1$.

$f_{T}: \quad$ Temperature dependence factor $=1$.

$f_{E}$ : Flow evolution factor

$\mathrm{Eq}$

Internal body conditions do not have considerable variation with respect to environmental pressure and temperature [8].

$$
\begin{aligned}
& f_{E}=1 / 2\left[\left(A+(1-A) e^{-\varepsilon_{M} / E_{1}}\right)+(B+(1-\right. \\
& \left.\left.B) e^{-\varepsilon_{M} / E_{2}}\right)\right]
\end{aligned}
$$

Where:

$A$ : Final value of flow evolution factor for exponential

1

$\varepsilon_{M}: \quad$ Effective Mises plastic strain.

$E_{1}$ : Characteristic transition strain for exponential 1.

$B$ : Final value of flow evolution factor for exponential

2.

$E_{2}$ : Characteristic transition strain for exponential 2.

2.3 Mechanical behavior of phalanx and polymer device. The main goal of this work was to analyze the flexural and tensile behavior of a fractured metacarpal joined by a medical device and two screws through finite element software. The assembly compound of the metacarpal and biopolymer device is presented in Fig., which depicts the join mechanism (Fig. .a) and the configuration of the loads (Fig. .b). All devices were set as PLLA. The biomedical device was a rectangular piece of $4 \mathrm{~mm}$ width, $16 \mathrm{~mm}$ length, and $1 \mathrm{~mm}$ thickness, and rounded edges with a $2 \mathrm{~mm}$ radius. It had two holes of $1.6 \mathrm{~mm}$ diameter and $12 \mathrm{~mm}$ distance between centers. Screws had $1.6 \mathrm{~mm}$ diameter, $4.5 \mathrm{~mm}$ length and $0.3 \mathrm{~mm}$ pitch. The head had $9.4 \mathrm{~mm}$ diameter and $1.2 \mathrm{~mm}$ length. The phalanx and the biomedical device were modeled with a Computer Aided Design software named Autodesk Inventor and the final result is shown in Fig. . The phalanx model developed by Holzbaur et al. (2005) [9] on the OpenSim platform was used [10]. After modeling, the assembly was exported to Ansys ${ }^{\circledR}$ Workbench software and the finite element analysis was carried out.
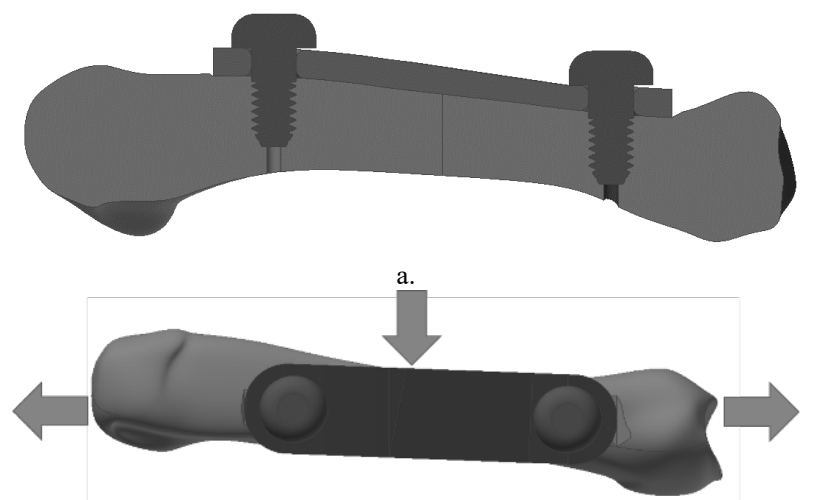

b.

Fig. 4. Metacarpal and PLLA device assembly. a. Cutting view of assembly and $\mathrm{b}$. Forces applied under the assembly

\subsection{Finite element analysis}

Finite element analyses of a tensile and flexural performance were developed. The metacarpal modulus was $16 \mathrm{GPa}$, with a Poisson's ratio of 0.3 , tensile yield strength of $80 \mathrm{MPa}$, and compressive yield strength of $117 \mathrm{MPa}$ [11]. Flexural 
performance simulation was implemented fixing the two ends of the metacarpal and applied a force aligned with the fracture. On the other hand, tensile performance was developed applying two loads with the same magnitude and opposite directions (Fig. .b). The applied loads are depicted in Table 1. Once the parameters were obtained, mechanical performance of fractured metacarpal joined with the PLLA device were determined. Former studies have demonstrated the relevance of the mesh density on the response of the finite element analysis [12]. Thus, the appropriate element size was determined and all simulations were carried out modifying the element size searching for response convergence. The mesh density was varied four times and were named as Types in Table 2. Thread surfaces, contact surface between device and bone and fractured surface were defined as Refinement surfaces in

Table 2 and the rest of the surfaces were defined as Regular surfaces (

Table ). Both groups of surfaces (Refinement surfaces and Regular surfaces) had a different size of elements.

Table 1. Load configurations for flexural and tensile analysis.

\begin{tabular}{c|c|c|c|c|c|c|c|c}
\hline $\begin{array}{c}\text { Time } \\
\text { (s) }\end{array}$ & \multicolumn{3}{|c|}{$\begin{array}{c}\text { Load } \\
\text { (N) }\end{array}$} & \multicolumn{4}{c}{$\begin{array}{c}\text { Toad } \\
\text { (N) }\end{array}$} \\
\hline 0 & 0 & 0 & 0 & 0 & 0 & 0 & 0 & 0 \\
0.3 & 20 & 30 & 60 & 100 & 3 & 4.5 & 9 & 15 \\
1 & 20 & 30 & 60 & 100 & 10 & 15 & 30 & 50 \\
\hline
\end{tabular}

Table 2. Types of mesh densities

\begin{tabular}{|c|c|c|}
\hline \multirow[b]{2}{*}{ Type } & Refinement surfaces & Regular surfaces \\
\hline & $\begin{array}{c}\text { Mean element size } \\
(\mathrm{mm})\end{array}$ & $\begin{array}{c}\text { Mean element size } \\
(\mathbf{m m})\end{array}$ \\
\hline I & 0.6 & 0.3 \\
\hline II & 0.3 & 0.15 \\
\hline III & 0.15 & 0.08 \\
\hline IV & 0.12 & 0.06 \\
\hline
\end{tabular}

Von Mises stress was analyzed in all simulations looking for elastic limits of assembly. All simulations were carried out taking into consideration large deflections owing the variances in stiffness due to changes in shape of the assembly parts.

\section{Results and Discussion}

The RMSE served to evaluate the difference between predicted and the observed value, the chosen rheological model had $98 \%$ accuracy, the parameter values were defined as presented in Table and agreed with a previous work [7]. Once the parameters of two networks were defined, a comparison between experimental behavior and the mathematical model was carried out and depicted in Fig. .

The rheological model based on Parallel Network demonstrated a correct prediction (Fig. ). The accuracy depended on the Neo-Hookean hyperelasticity Network (first Network) parallel with a Neo-Hookean hyperelasticity with a power law flow (second Network). Based on these results, the first Network exposed the hyperelasticy related with intermolecular forces and the second Network described the intramolecular resistance and its performance taking into account the flow. Accordingly, this model decomposed total stress into an elastic and history dependent component in good agreement with prior studies $[4,13]$. These results are in concordance with the theory [7.13-14].

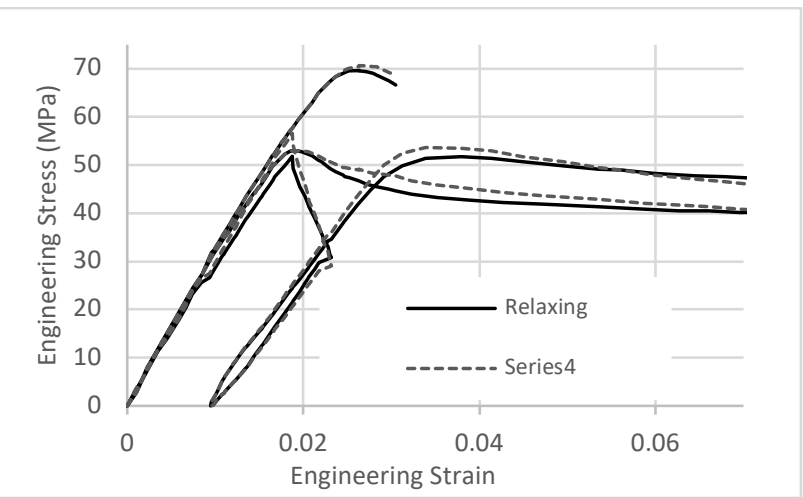

Fig. 5. PLLA Tensile behavior varying strain rate and relaxation. Comparison between experimental data and prediction data

The mathematical model evidenced that intramolecular flow resistance related to shear modulus is almost 15 times higher than the intermolecular resistance related to shear stress. This relation demonstrated that Van der Waals forces are not effective to maintain the polymeric chains joined under a load, besides the covalent bonds are responsible for the main strength [14].

Figure 5 demonstrated that prediction of the mechanical behavior can accurately determine the ultimate stress. Moreover, the elastic modulus was correctly predicted as well as the fracture stress defined.

In tensile test with strain rate of $0.001 / \mathrm{s}$ there is a gap between ultimate stress and stress at $0.049 \mathrm{~mm} / \mathrm{mm}$. This difference between predicted and experimental behavior is owing to the flow evolution factor, which related the plastic strain with flow response.

Relaxation behavior also exhibited this difference at stress related to $0.031 \mathrm{~mm} / \mathrm{mm}$ [15]. The relation between useful stress and the strength depended only upon the slope of the load; it depended on the combination between the load time and slope instead. Although some loads have similar slopes, the bearing of assembly was lesser when the load was applied during a longer period of time because of the intramolecular strength [16].

Since mesh density has a direct influence in the accuracy of response, a relation between element size and convergence was accomplished. All simulations were developed with the four types of meshing size (

Table 3). For instance, Fig 6 depicted the flexural response at $100 \mathrm{~N}$ with different meshing types.

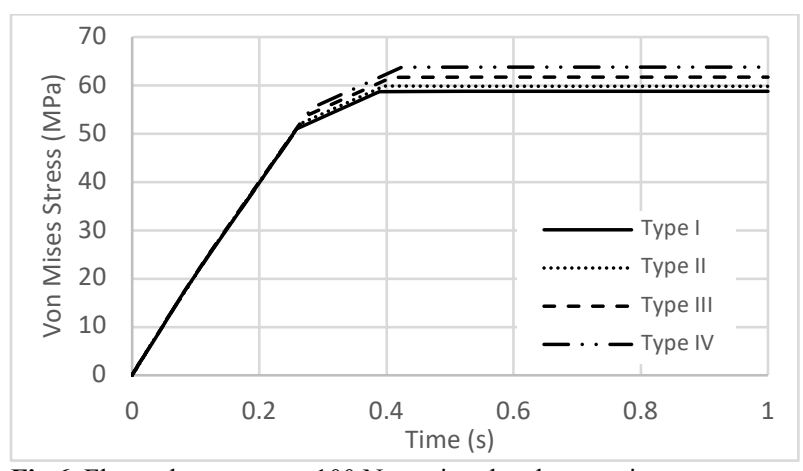

Fig 6. Flexural response at $100 \mathrm{~N}$ varying the element size

The response difference between Type III and Type IV was less than $1 \%$ in all simulations. Decrease of element size generated a response convergence but sacrificed computing resources and time [12]. 
The response analysis and comparison varying the load in flexural and tensile behavior studies were carried out. Fig. depicts Von Mises stress generated in all assembly under flexural loads. Due to the type of loads, the assembly does not reach the yield point. Nevertheless, there exists the possibility that a constant increase of load throughout time forces the assembly to reach the plastic stress. Considering this, the tensile response with different loads with constant slope was analyzed.

Fig. 8. illustrates the tensile response. Von Mises stress was depicted until $80 \mathrm{MPa}$ owing to this is the yield stress of bone. Yield stress of bone was chosen as a limit because the maximum stress is located in bone holes. Von Mises stress is almost proportional to load. The higher the load, the shorter the strength time.

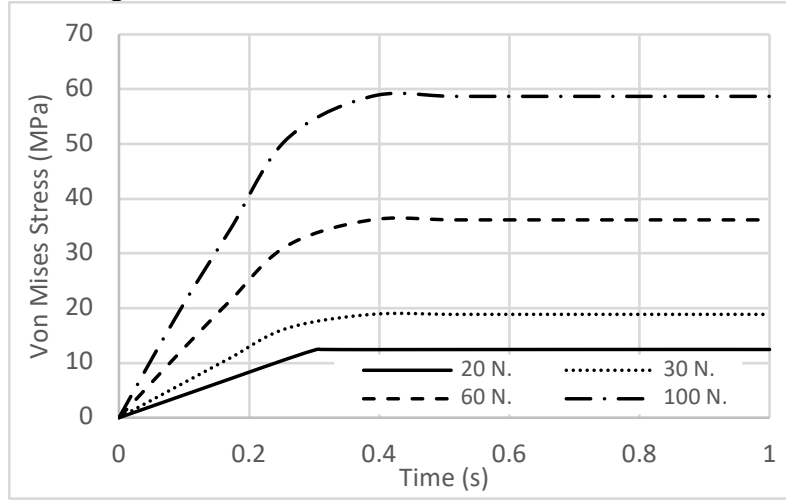

Fig. 7. Assembly flexural response with Type IV element size configuration.

In tensile behavior as well as in flexural behavior maximum stresses were around the holes in the metacarpal. These results agreed with literature $[17,18]$ which suggested that defects on structures such as geometry changes, cracks and holes increment stress and generate failure. Nevertheless, the Von Mises stresses of polymer devices were below that yield stress in all simulations and highest loads were borne by bone, which complied with prior studies which described the mechanical performance advantages of PLLA devices under regular hand loads [5] and suggest that stress concentration in the main cause of failure in this kind of fixation method. This concurs with prior work [7] in which pines were used instead of screws and similar stress concentrations were obtained. Nevertheless, the use of screws incremented the strength of fixation.

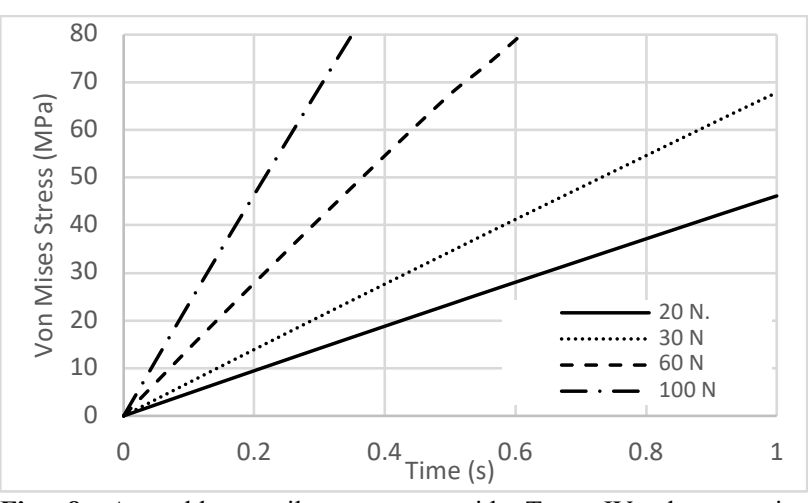

Fig. 8. Assembly tensile response with Type IV element size configuration.

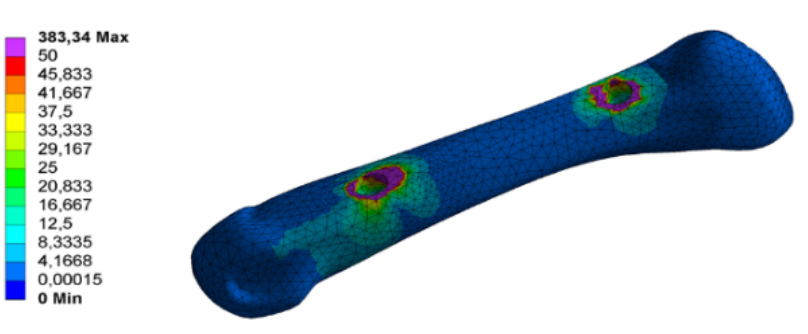

Fig. 9. Equivalent Von Mises stress in phalanx.

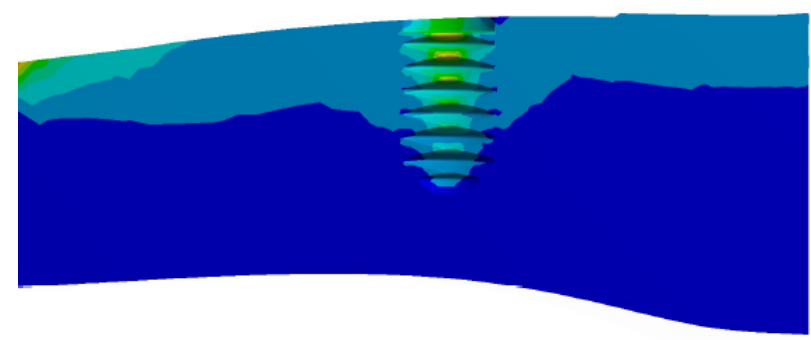

Fig. 20. Equivalent Von Mises stress of threaded hole

Table 3. Obtained Parameters of PLLA based on Parallel Network Model.

\begin{tabular}{c|c|c|c|c|c|c|c|c|c|c|c}
\hline \multicolumn{10}{c|}{ Network 1 } & \multicolumn{10}{c}{ Network 2 } \\
\hline $\boldsymbol{\mu}$ & $\boldsymbol{\kappa}$ & $\boldsymbol{\mu}$ & $\boldsymbol{\kappa}$ & $\boldsymbol{R}_{\boldsymbol{\tau}}$ & $\boldsymbol{m}$ & $\boldsymbol{f}_{\boldsymbol{p}}$ & $\boldsymbol{f}_{\boldsymbol{T}}$ & $\boldsymbol{A}$ & $\boldsymbol{E}_{\boldsymbol{1}}$ & $\boldsymbol{B}$ & $\boldsymbol{E}_{\boldsymbol{2}}$ \\
\hline$(\mathrm{MPa})$ & $(\mathrm{MPa})$ & $(\mathrm{MPa})$ & $(\mathrm{MPa})$ & $(\mathrm{MPa})$ & - & - & - & - & - & - & - \\
60.251 & 5191.040 & 1009.470 & 5920.130 & 69.316 & 13.825 & 1.000 & 1.000 & 0.016 & 0.032 & -0.016 & 9.580 \\
\hline
\end{tabular}

Where: - = dimensionless.

\section{Conclusions}

Intramolecular and intermolecular incidence on mechanical behavior of PLLA were described accurately with a Parallel Network Model. The inference of flow response, strain rate, hyperelasticity, temperature, and relaxation response were included in the viscoplastic model. Furthermore, flow response at plastic strains is influenced for flow evolution factor. The mesh density factor is related with simulation response convergence. In addition, internal conditions of the limb do not have suitable effects on mechanical behavior of PLLA device compared to its behavior in environmental conditions. The load, load rate, and the time are related to the strength of the device. Once the analysis was accomplished, in order to use a biopolymer plate and screws as a method to repair fracture phalanx, it is necessary to focus on some conditions. The use of this kind of configuration has to consider stress concentration owing to thread holes in the bones. PLLA has adequate strength to bear tensional and flexural loads related to daily activities. Mathematical models which describes mechanical behavior of polymers, if applied properly, can provide great insight into the performance devices [4]. Future analysis should include reinforced fiber inside polymer, anisotropic response, and degradation of materials.

This is an Open Access article distributed under the terms of the Creative Commons Attribution License 


\section{References}

1. Diaz-Garcia, R., Waljee, J. F., 2013. Current Management of Metacarpal Fractures. J. Hand Clin. 29, 507-518.

2. Agarwal, R., Garcia, A. J., 2015. Biomaterial strategies for engineering implants for enhanced osseointegration and bone repair. J. Adv. Drug Deliv. Rev. 94, 53-62.

3. Avilés, O. F. S., Mauledoux, M. F. M., Rubiano, O. G. M., Ramirez, H. F. G., Dutra, M. S., 2016. FEA of Bioabsorbable Material to Repair Hand Fractures. J. Appl. Mech. Mater. 823, 173178.

4. Bergström, J. S., Hayman, D., 2016. An Overview of Mechanical Properties and Material Modeling of Polylactide (PLA) for Medical Applications. J. Ann. Biomed. Eng. 44, 330-340.

5. Narayanan, G., Vernekar, V. N., Kuyinu, E. L., Laurencin, C. T., 2016. Poly (lactic acid)-based biomaterials for orthopaedic regenerative engineering. J. Adv. Drug Deliv. Rev. 107, 247-276.

6. Onuma, Y., Serruys, P. W., 2011. Bioresorbable Scaffold: The Advent of a New Era in Percutaneous Coronary and Peripheral Revascularization?. J. Circulation. 123, 779-797.

7. Nunez, D.A., Bernal, A., Mauledoux , M.F., Aviles, O.F., 2017. Analysis of Mechanical Behavior of a Device Made of Poly L Lactide Acid for Reconstruction of Phalanx Fracture. J. Chem. Eng. Trans. 57, 1357-1362.

8. Kasper, D. L., Fauci, A. S., Hauser, S., Longo, D., Jameson, J. L., Loscalzo, J., 2016. Harrisons Manual of Medicine. 19th ed. McGraw-Hill Education.
9. Holzbaur, K. R. S., W. M. Murray., Delp, S. L., 2005. A Model of the Upper Extremity for Simulating Musculoskeletal Surgery and Analyzing Neuromuscular Control. J. Ann. Biomed. Eng. 33, 829840 .

10. Delp, S. L., Anderson, F. C., Arnold, A. S., Loan, P., Habib, A., John, C. T., Guendelman, E., Thelen, D. G., 2007. OpenSim: Open-Source Software to Create and Analyze Dynamic Simulations of Movement. J. IEEE Trans. Biomed. Eng. 54, 1940-1950.

11. Enderle , J., Bronzino, J., 2011. Introduction to Biomedical Engineering. Elsevier Science.

12. Liu, Y., Glass, G., 2013. Effects of Mesh Density on Finite Element Analysis. SAE Technical Paper. 2013-01-1375.

13. Bergstrom, J. S., 2015. Mechanics of solid polymers: theory and computational modeling. William Andrew.

14. Bergström, J., 2015. Viscoplasticity Models. J. Mechanics of Solid Polymers. 371-436.

15. Bergstrom, J. S., 2012. PolyUMod--A Library of Advanced User Materials. J. Veryst Eng. LLC.

16. Van Krevelen , D. W., Te Nijenhuis , K., 2009. Mechanical Properties of Solid Polymers. J. Properties of Polymers. Fourth ed. Elsevier.

17. Trojan, F., 1986. Engineering Materials and Their Applications. Third ed. Jaico Publishing House, New York.

18. Budynas, R. G., Nisbett, J. K., 2014. Shigley's Mechanical Engineering Design. McGraw-Hill Education. 